\title{
Accurate microfour-point probe sheet resistance measurements on small samples
}

Thorsteinsson, Sune; Wang, Fei; Petersen, Dirch Hjorth; Hansen, Torben Mikael; Kjær, Daniel; Lin, Rong; Kim, Jang-Yong; Nielsen, Peter Folmer; Hansen, Ole

Published in:

Review of Scientific Instruments

Link to article, DOI:

$10.1063 / 1.3125050$

Publication date:

2009

Document Version

Publisher's PDF, also known as Version of record

Link back to DTU Orbit

Citation (APA):

Thorsteinsson, S., Wang, F., Petersen, D. H., Hansen, T. M., Kjær, D., Lin, R., Kim, J-Y., Nielsen, P. F., \& Hansen, O. (2009). Accurate microfour-point probe sheet resistance measurements on small samples. Review of Scientific Instruments, 80(5), 053902. https://doi.org/10.1063/1.3125050

\section{General rights}

Copyright and moral rights for the publications made accessible in the public portal are retained by the authors and/or other copyright owners and it is a condition of accessing publications that users recognise and abide by the legal requirements associated with these rights.

- Users may download and print one copy of any publication from the public portal for the purpose of private study or research.

- You may not further distribute the material or use it for any profit-making activity or commercial gain

- You may freely distribute the URL identifying the publication in the public portal

If you believe that this document breaches copyright please contact us providing details, and we will remove access to the work immediately and investigate your claim 


\title{
Accurate microfour-point probe sheet resistance measurements on small samples
}

\author{
Sune Thorsteinsson, ${ }^{1}$ Fei Wang, ${ }^{2}$ Dirch $\mathrm{H}$. Petersen, ${ }^{1,2}$ Torben Mikael Hansen, ${ }^{2}$ \\ Daniel Kjær, ${ }^{1}$ Rong Lin, ${ }^{1}$ Jang-Yong Kim, ${ }^{2}$ Peter F. Nielsen, ${ }^{1}$ and Ole Hansen ${ }^{2,3, a)}$ \\ ${ }_{1}^{1}$ Capres A/S, Scion-DTU, Building 373, DK-2800, Kgs. Lyngby, Denmark \\ ${ }^{2}$ Department of Micro- and Nanotechnology, Technical University of Denmark, DTU Nanotech Building \\ 345 East, DK-2800, Kgs. Lyngby, Denmark \\ ${ }^{3}$ Danish National Research Foundation's Center for Individual Nanoparticle Functionality (CINF), \\ Technical University of Denmark, DK-2800, Kgs. Lyngby, Denmark
}

(Received 23 February 2009; accepted 6 April 2009; published online 1 May 2009)

\begin{abstract}
We show that accurate sheet resistance measurements on small samples may be performed using microfour-point probes without applying correction factors. Using dual configuration measurements, the sheet resistance may be extracted with high accuracy when the microfour-point probes are in proximity of a mirror plane on small samples with dimensions of a few times the probe pitch. We calculate theoretically the size of the "sweet spot," where sufficiently accurate sheet resistances result and show that even for very small samples it is feasible to do correction free extraction of the sheet resistance with sufficient accuracy. As an example, the sheet resistance of a $40 \mu \mathrm{m}(50 \mu \mathrm{m})$ square sample may be characterized with an accuracy of $0.3 \%(0.1 \%)$ using a $10 \mu \mathrm{m}$ pitch microfour-point probe and assuming a probe alignment accuracy of $\pm 2.5 \mu \mathrm{m}$. (C) 2009 American Institute of Physics. [DOI: 10.1063/1.3125050]
\end{abstract}

\section{INTRODUCTION}

In a wide range of semiconductor applications sheet resistance of the thin films used is of prime importance for the final device performance. It follows that experimental characterization of thin film sheet resistance is essential. For decades four-point probe measurements have been the preferred metrology technique ${ }^{1,2}$ due to the low requirements on sample preparation and the high accuracy. The measurements are mostly done on large samples-test-wafers-with dimensions much larger than the pitch of the four-point probes. With the increasing wafer size and processing costs, considerable savings may be realized if accurate sheet resistance characterization could be done on small dedicated areas on device wafers instead.

Recently microfabricated four-point probes ${ }^{3}$ have become commercially available. ${ }^{4}$ Microfour-point probes (M4PPs) have been proven to be a very useful tool for characterizing ultrashallow junctions without junction penetration, ${ }^{5}$ and resolving stitching phenomena on laser annealed junctions. ${ }^{6,7}$ Recently even scanning Hall mobility measurements have been demonstrated ${ }^{8,9}$ using M4PPs. The available M4PPs have a small pitch that allows for measurements on scribe line test pads of production wafers. ${ }^{6}$

Four-point probe measurements on samples with dimensions of the same scale as the probe pitch, however, are strongly affected by the proximity of insulating sample boundaries, thus interpretation of the measurement results is nontrivial. Geometric correction factors for the proximity of sample boundaries have been calculated for a variety of different sample geometries ${ }^{10-17}$ including single boundaries,

${ }^{a)}$ Electronic mail: ole.hansen@nanotech.dtu.dk. corners, squares, rectangles, and circles; even finite thickness samples ${ }^{18}$ have been treated. In some cases analytical correction factors exist, however, in the presence of boundary effects, from two or more boundaries, the analytical expressions are complicated and require accurate knowledge about sample geometry and probe position; this detailed knowledge is rarely available.

Measurements using dual configurations, in which two of three different, nontrivial permutations of voltage and current pins are used to extract the sheet resistance of a sample, have been proven to correct in-line positional errors, ${ }^{15,19-21}$ and to extract the correct sheet resistance regardless of sample shape when certain symmetries exist. ${ }^{15,19}$ The dual configuration method is known to significantly decrease the effect of the boundary for circular samples, provided that the probe is located more than a few times the electrode pitch from the edge. ${ }^{15,20}$ Further, it is expected to have a similar effect on other samples. ${ }^{21}$ Even though the method is based on a thin sample assumption, it has been shown to give accurate sheet resistances even on samples with a thickness of the order of the electrode pitch. ${ }^{22}$

Here we explore the use of dual configuration M4PP measurements for correction free, accurate characterization of small samples with dimensions on the order of a few times the probe pin pitch. We analyze the requirements on probe positioning to achieve this goal on several simple sample geometries: single boundary, double boundary, circular disks, squares, and rectangles. We show that with dual configuration M4PP measurements even significantly smaller samples than those suggested in Ref. 6 may be accurately characterized without applying correction factors. Finally, we demonstrate practical measurements on small square samples. 

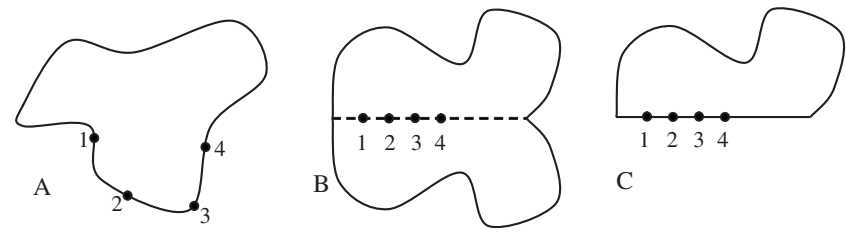

FIG. 1. Schematic of four-point probe measurement cases. In (A) the case discussed by van der Pauw is shown. In (B) a collinear four-point probe measurement on the trace of the sample mirror plane is shown. In (C) a van der Pauw measurement on half of the symmetric sample in (B) is shown.

\section{THEORY}

Accurate measurement of the sheet resistance $R_{\square}$ of a small sample may in theory be achieved by dual configuration four-point probe measurements on the perimeter of any small sample, since van der Pauw ${ }^{23,24}$ has shown that for any filamentary sample

$$
\exp \left(-\frac{\pi R_{a}}{R_{\square}}\right)+\exp \left(-\frac{\pi R_{c}}{R_{\square}}\right)=1
$$

or

$$
\exp \left(\frac{\pi R_{a}}{R_{\square}}\right)-\exp \left(\frac{\pi R_{b}}{R_{\square}}\right)=1,
$$

where $R_{a}=V_{23} / I_{14}, R_{b}=V_{24} / I_{13}$, and $R_{c}=V_{43} / I_{12}$, respectively are the measured resistances with the four probe pins (1-4) at an arbitrary but fixed position on the perimeter of the sample as illustrated in Fig. 1(a). Here $I_{i j}$ is the current forced through the sample from probe pin $i$ to probe pin $j$ while $V_{k \ell}$ is the voltage measured between probes $k$ and $\ell$. However, in real measurements it is not convenient or possible to position the probe pins on the perimeter, due to alignment problems, possible damage to the probes, curved sample perimeter, or ill defined sample perimeter.

For a small sample with one or more mirror planes, however, four-point resistance measurements on the trace of a mirror plane using a collinear four-point probe [see Fig. 1(b)] lead to a vanishing current density $\mathbf{J}$ normal to the mirror plane, $\mathbf{J} \cdot \mathbf{n}=0$, where $\mathbf{n}$ is a unit vector normal to the mirror plane. Thus the potential is unaffected if the mirror plane is replaced by an insulating boundary in this case, and the measured resistances $R_{A}=V_{23} / I_{14}, R_{B}=V_{24} / I_{13}$, and $R_{C}=V_{43} / I_{12}$ are exactly half of the resistances one would measure on the perimeter of half the sample with the probes in the same position on the boundary [see Fig. 1(c)], that is $R_{A}=R_{a} / 2$, $R_{B}=R_{b} / 2$, and $R_{C}=R_{c} / 2$, and thus the resistances fulfill

$$
\exp \left(-\frac{2 \pi R_{A}}{R_{\square}}\right)+\exp \left(-\frac{2 \pi R_{C}}{R_{\square}}\right)=1
$$

or

$$
\exp \left(\frac{2 \pi R_{A}}{R_{\square}}\right)-\exp \left(\frac{2 \pi R_{B}}{R_{\square}}\right)=1,
$$

for an arbitrary but fixed probe arrangement on the trace of the mirror plane. Extraction of the sheet resistance from a pair of resistance measurements would thus also simultaneously correct for unintended errors in probe pin spacing. ${ }^{19}$

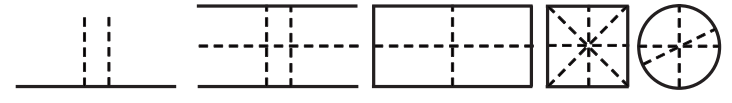

FIG. 2. Schematic of some simple sample geometries with mirror planes (dashed lines).

In practical measurements it may not be possible to place the probes exactly on the trace of the mirror plane thus the conditions for use of Eq. (2) are violated. The resistances, however, will fulfill

$$
\exp \left(-\frac{2 \pi R_{A}}{\chi R_{\square}}\right)+\exp \left(-\frac{2 \pi R_{C}}{\chi R_{\square}}\right)=1
$$

or

$$
\exp \left(\frac{2 \pi R_{A}}{\chi R_{\square}}\right)-\exp \left(\frac{2 \pi R_{B}}{\chi R_{\square}}\right)=1,
$$

where $\chi$ is a parameter $0<\chi \leq 2$. In a rather wide region near the mirror plane, however, $\chi \simeq 1$ and thus quite accurate sheet resistance estimates $R_{\square \text { est }}=\chi R_{\square}$ may be extracted from measured dual configuration four-point resistances using Eq. (2). The resulting relative error on the extracted sheet resistance is

$$
\left(R_{\square \text { est }}-R_{\square}\right) / R_{\square}=\chi-1,
$$

thus $\chi$ serves as an error parameter for this approach.

\section{ANALYTIC, NUMERIC, AND EXPERIMENTAL METHODS}

For the simple geometries, as illustrated in Fig. 2, with a few boundaries: the single straight boundary, the corner, the narrow stripe, and the circular disk, analytic expressions for the four-point resistances $R_{A}$ and $R_{B}$ are evaluated for varying probe position and orientation, and subsequently Eq. (3) is solved numerically for $\chi$ with each pair of four-point resistances. These analytic expressions are all based on a point current source model for the single straight boundary, ${ }^{9}$ which by use of conformal mapping ${ }^{25}$ may also be applied to other geometries such as the corner, the narrow stripe, and the circular disk (see Appendix).

For rectangles and squares, both analytic expressions (double infinite sums of point source solutions ${ }^{9}$ ) and finite element modeling using COMSOL MULTIPHYSICS 3.3 are used for evaluation of the four-point resistances, whereafter Eq. (3) is solved numerically for $\chi$ with each pair of fourpoint resistances. A comparison of the results from the two techniques is used to validate convergence and accuracy.

The M4PP measurements were performed using a CAPRES microRSP-M150 system. ${ }^{4}$ The M4PP used in these experiments consists of $\mathrm{Ni}$ coated silicon cantilever electrodes extending from the edge of a silicon die; in the experiments probes with a probe pin pitch of $10 \mu \mathrm{m}$ were used. The sample used was a patterned shallow $(80 \mathrm{~nm})$ p-type junction formed in Ge using Rapid Thermal Annealing (RTA) of a boron implant $\left(10 \mathrm{keV}, 2 \times 10^{15} \mathrm{~cm}^{-2}\right)$ following a preamorphization implant. 


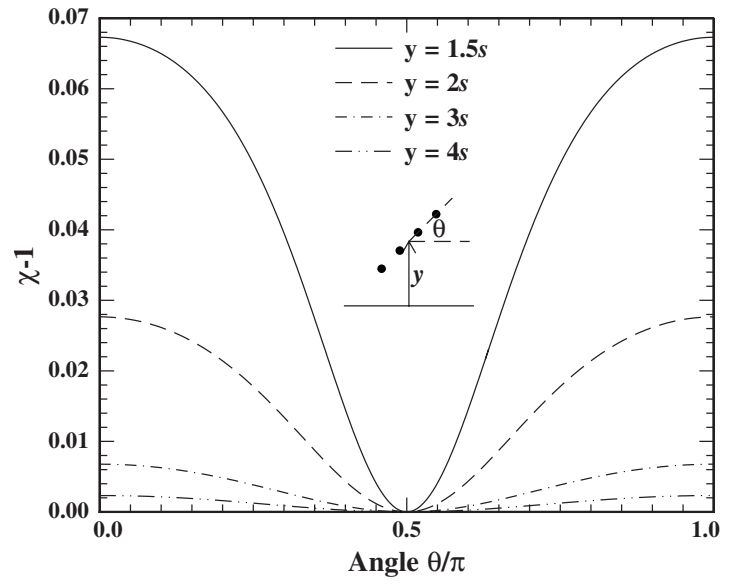

FIG. 3. The error $\chi-1$ as a function of angle $\theta$ between the line of the probe and a single insulating boundary with the distance between the boundary and the probe center $y$ as parameter. Calculations for $y / s$ $\in[3 / 2,2,3,4]$ are shown.

\section{RESULTS AND DISCUSSION}

Dual configuration sheet resistance measurements on infinitely large homogenous samples may be performed with a repeatability better than $0.1 \%$ (Ref. 26) using M4PPs since Eq. (2) corrects for in-line position errors and only small off-line position errors contribute. It would be desirable if measurements on small samples could be performed with approximately the same accuracy, such that the presence of the sample boundaries contributes with an error less than $0.1 \%$ to make the total error less than $\sim 0.15 \%$. In many practical applications, however, an accuracy of $0.3 \%$ is sufficient, thus $|\chi-1| \leq 0.3 \%$ may be allowed; this condition is therefore used in the discussion below while graphs also illustrate the effect of enforcing the condition $|\chi-1| \leq 0.1 \%$. The proper length scale for the structures investigated here is the probe pin pitch $s$, therefore all dimensions are stated in units of $s$.

\section{A. Single insulating boundary sample}

A thin semi-infinite sample with a single insulating straight boundary has mirror planes with traces normal to the boundary, and even though it does not qualify as a small sample an analysis may be helpful in interpreting the behavior of more complicated structures. From the presence of a mirror plane it follows that any dual configuration four-point probe measurement with the line of the probe normal to the boundary will fulfill Eq. (2), thus $\chi \equiv 1$ and accurate sheet resistance extraction results. Now, in real measurements the probe may be rotated some small angle $\Delta \theta$ away from the ideal angle $\theta=\pi / 2$ between probe and boundary and a small error in the extracted sheet resistance results.

For this sample comparatively simple analytic expressions for the resistances $R_{A}, R_{B}$, and $R_{C}$ as a function of probe position and orientation are easily obtained using mirror images. Even in this simple sample, however, $\chi$ can only be calculated numerically by solving Eq. (3) for $\chi$. In Fig. 3 such calculations are shown for probe center distances $y$ $\in[3 s / 2,2 s, 3 s, 4 s]$ from the insulating boundary as a function of the angle $\theta$ between the line of the probe and the

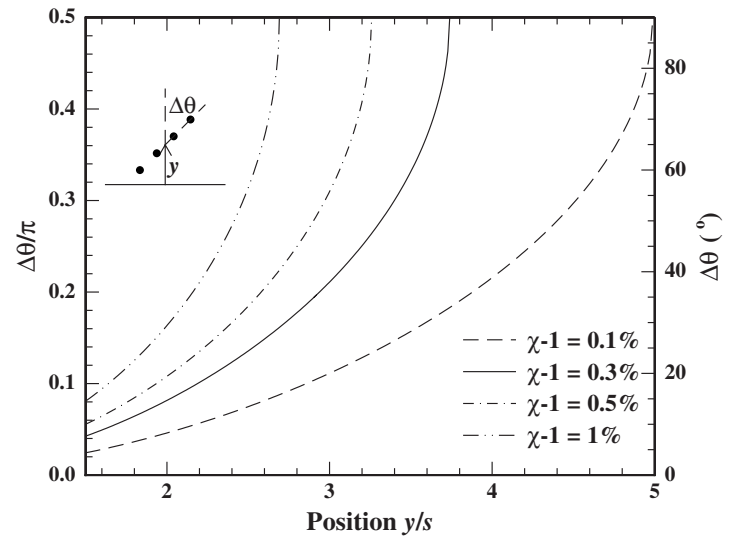

FIG. 4. Allowable misalignment angle $\Delta \theta$ as a function of the probe to boundary distance $y$, with the error $\chi-1$ as parameter for measurements on a sample with a single straight insulating boundary. Calculations for $\chi-1$ $\in[0.1 \%, 0.3 \%, 0.5 \%, 1 \%]$ are shown.

boundary. Here $y / s=3 / 2$ is the minimum relevant probe center to boundary distance since in the ideal configuration $\theta$ $=\pi / 2$ one probe is exactly on the boundary.

In Fig. 4 the allowable angle alignment error $\Delta \theta$ from the ideal probe angle $\theta=\pi / 2$ is shown as a function of probe to boundary separation with the resulting error contribution $\chi-1$ as parameter. Calculations for $\chi-1$ $\in[0.1 \%, 0.3 \%, 0.5 \%, 1 \%]$ are shown. In particular, the full line shows the allowable angle alignment error $\Delta \theta$ at $\chi$ $-1=0.3 \%$; obviously, for probe-boundary distances larger than $\sim 3.75 s$ the probe-boundary angle is unimportant for the resulting error in this case, and thus at a distance of approximately $3.75 s$ the measurement is almost unaffected by the presence of the boundary. Further, it can be seen that even in closer proximity of the edge, $y \geq 3 s / 2$, angle alignment errors as large as approximately $\pm 7^{\circ}$ may be allowed.

\section{B. Corner with top angle $\varphi$}

A semi-infinite region with two straight insulating boundaries intersecting at an angle $\varphi$ to form a corner has a mirror plane, with the bisector as the trace of the mirror plane. This problem may be solved using the method of images, however, part of the behavior may be analyzed easily by recognizing that the conformal mapping $z=w^{\pi / \varphi}$ maps the corner on a single straight boundary. A radially aligned equispaced collinear probe at the angle $\theta$ is mapped on a radially aligned collinear probe at the angle $\pi \theta / \varphi$, which however is not equispaced. In particular the bisector is mapped on the straight boundary mirror plane. Since the mapped probes are not equispaced the length scale of the problem is modified, but Eq. (3) may still be used and with the probes on the mirror plane, Eq. (2) is still exact. We can however conclude that for a radially aligned probe the allowable angular misalignment is in worst case approximately $\pm(\varphi / \pi)$ $\times 7^{\circ}$ by a direct comparison to the single straight boundary analysis in the previous subsection.

Probes that are not radially aligned, however, is a more difficult problem since they are not mapped on a collinear probe. We shall not pursue that issue further. 


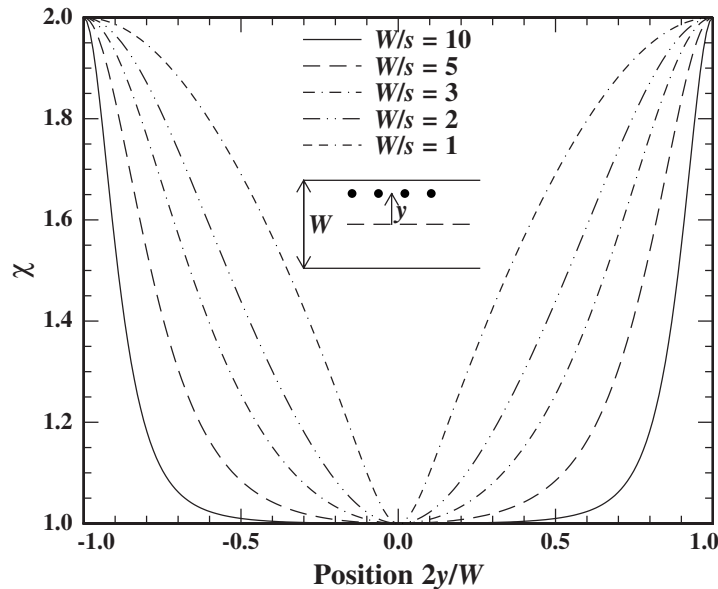

FIG. 5. Error parameter $\chi$ for a stripe of width $W$ with the probe parallel to the stripe as a function of probe displacement $y$ from the mirror plane. Calculations for normalized stripe widths $W / s \in[1,2,3,5,10]$ are shown.

\section{Narrow stripe sample}

An infinitely long narrow stripe of width $W$ has two types of mirror planes, one parallel to the insulating boundaries and an infinite number of mirror planes normal to the insulating boundaries. Analytic expressions for the four-point resistances $R_{A}, R_{B}$, and $R_{C}$ may be obtained using conformal mapping and the solution for a single straight boundary, as shown in the Appendix.

Figure 5 shows the error parameter $\chi$ for a stripe of width $W$ with the probe parallel to the stripe as a function of probe displacement $y$ from the mirror plane. Calculations for normalized stripe widths $W / s \in[1,2,3,5,10]$ are shown. From Fig. 5 it may be seen that for small widths of the stripe, the probe is allowed to move closer to the parallel boundary than in the single boundary case for a given allowed error, since the two boundaries tend to quench each other. We shall elaborate further on this behavior below.

In Fig. 6 the error parameter $\chi$ is shown as a function of the angle $\theta$ between the probe and the stripe boundary when the probe center is in the middle of the stripe. Calculations for $W / s \in[3,3.5,5,10]$ are shown. In all cases $\chi \leq 1$ and

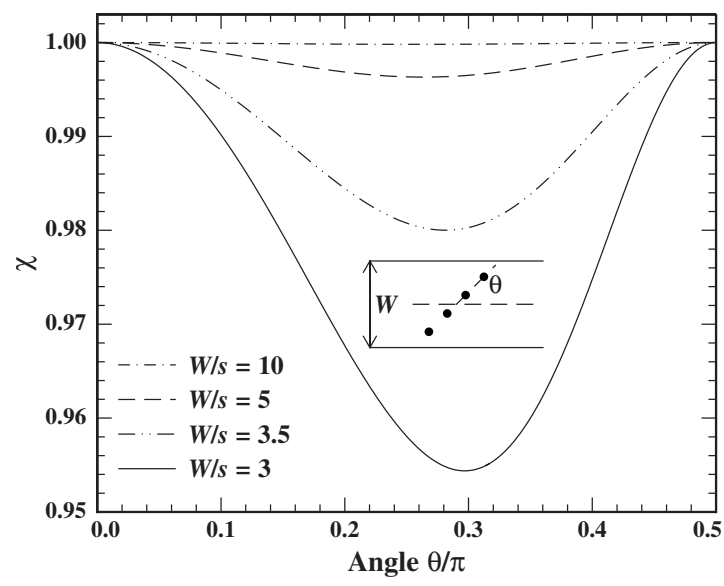

FIG. 6. Error parameter $\chi$ for a stripe of width $W$ with the probe center at $W / 2$ as a function of the angle $\theta$ between the probe and the insulating boundaries. Calculations for $W / s \in[3,3.5,5,10]$ are shown.

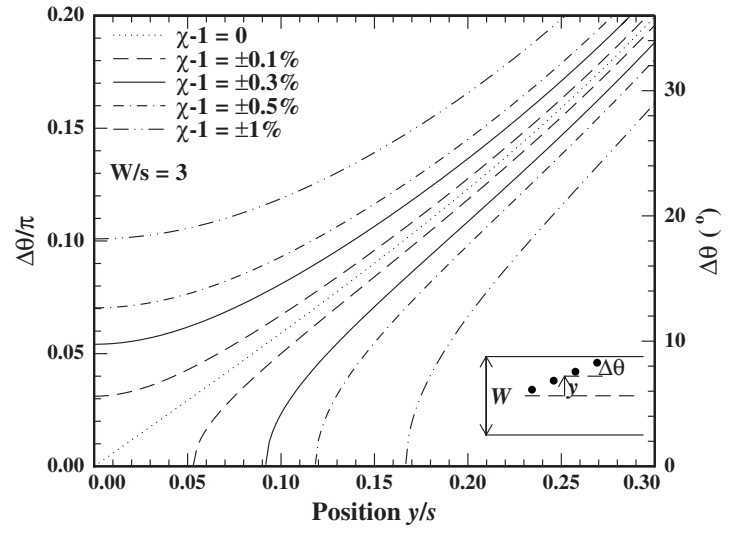

FIG. 7. Relation between allowable angular $(\Delta \theta)$ and lateral $(y)$ misalignments for a probe parallel to the boundaries of a stripe of width $W=3 \mathrm{~s}$. The dotted curve shows the trajectory where $\chi \equiv 1.00$. Calculations for $\chi-1$ $\in[ \pm 0.1 \%, \pm 0.3 \%, \pm 0.5 \%, \pm 1.0 \%]$ are shown.

the two mirror planes are easily recognized. Only for the narrow stripes $W=3 s$, where a full rotation is just possible, and $W=3.5 \mathrm{~s}$ a significant error due to rotation results. The allowable angular misalignment is significantly larger for a probe parallel to the boundaries than for a probe orthogonal to the boundaries.

In Fig. 7 constant error curves relating angular $(\Delta \theta)$ and lateral $(y)$ misalignment for a probe with an ideal position in the middle of the stripe parallel to the boundaries. The dotted curve shows the trajectory where $\chi=1.00$. Obviously, the effects of angular and lateral misalignments on the resulting error show a tendency to cancel each other. It follows, that evaluation of the individual allowable misalignments represents a worst case scenario.

In Fig. 8 the allowable angular misalignment $\Delta \theta$ as a function of stripe width $W$ is shown. The allowable angular misalignment increases rapidly with increasing sample width and with $W \geq 3 \mathrm{~s}$ it becomes larger than approximately $\pm 10^{\circ}$.

In Fig. 9 the allowable lateral misalignment $y$ is shown as a function of stripe width $W$ when the probe is parallel to the boundaries of the stripe. Calculations for $\chi-1$ $\in[0.1 \%, 0.3 \%, 0.5 \%, 1 \%]$ are shown. The allowable misalignment initially increases rapidly with increasing sample width and for large sample widths $W \geq 8 s$ it increases

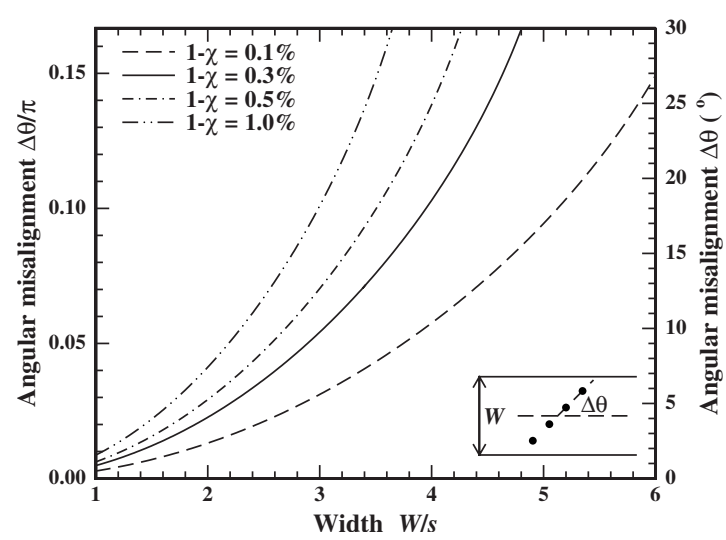

FIG. 8. Allowable angular misalignment $\Delta \theta$ as a function of stripe width $W$. Probe initially parallel to the boundaries and in the middle of the stripe. Calculations for $1-\chi \in[0.1 \%, 0.3 \%, 0.5 \%, 1 \%]$ are shown. 


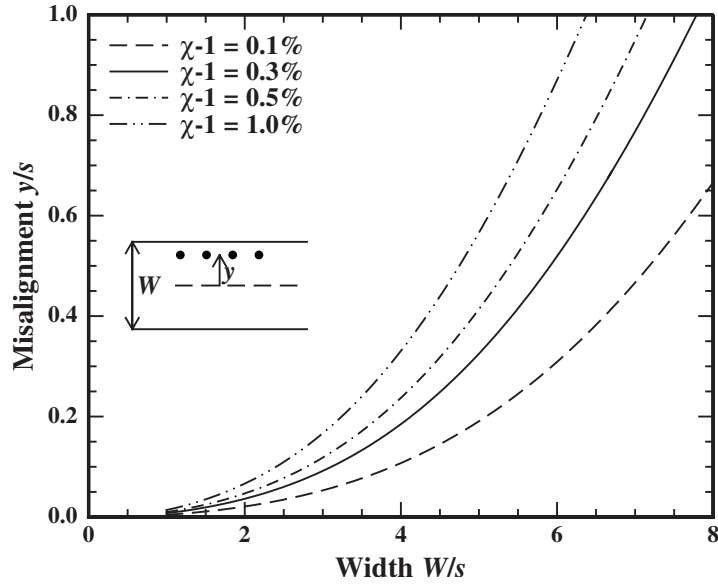

FIG. 9. Allowable lateral misalignment $y$ as a function of stripe width. Probe parallel to the boundary. Calculations for $\chi-1$ $\in[0.1 \%, 0.3 \%, 0.5 \%, 1 \%]$ are shown.

nearly linearly with sample width, such that a minimum distance $(\sim 3.75 \mathrm{~s})$ from a parallel boundary is required. In this regime the behavior is similar to that of two noninteracting boundaries, where a probe displaced more than $\sim 3.75 \mathrm{~s}$ from any of the boundaries remains unaffected by these. At small sample widths a smaller distance from the boundary is allowed.

\section{Circular disk samples}

The circular disk is comparatively simple to analyze since any diameter is a trace of a mirror plane. It follows that only a misalignment normal to the intended diagonal needs to be characterized.

Figure 10 shows isoerror contours (dashed and full black lines) for a circular disk of radius $R=5 \mathrm{~s}$ along with the trace (full blue line) of the probe center with one probe pin on the disk boundary. Isoerror contours for $\chi-1$

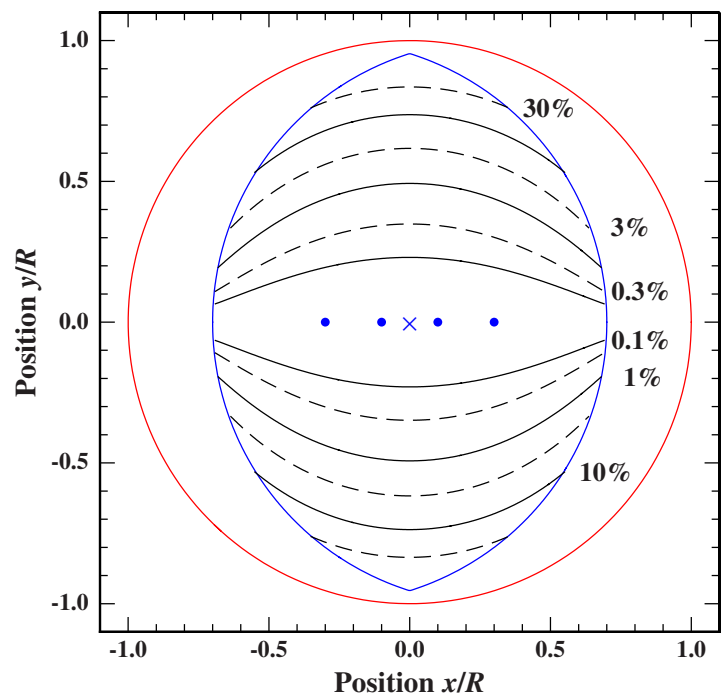

FIG. 10. (Color online) Isoerror contours on a circular disk with radius $R=5 \mathrm{~s}$. The full red curve indicates the sample perimeter, while the full blue curve is the trajectory of the center of the probe with one pin on the perimeter of the disk. Isoerror contours for $\chi-1$ $\in[0.1 \%, 0.3 \%, 1 \%, 3 \%, 10 \%, 30 \%]$ are shown. To indicate the scale a probe positioned exactly at the center of the disk is shown.

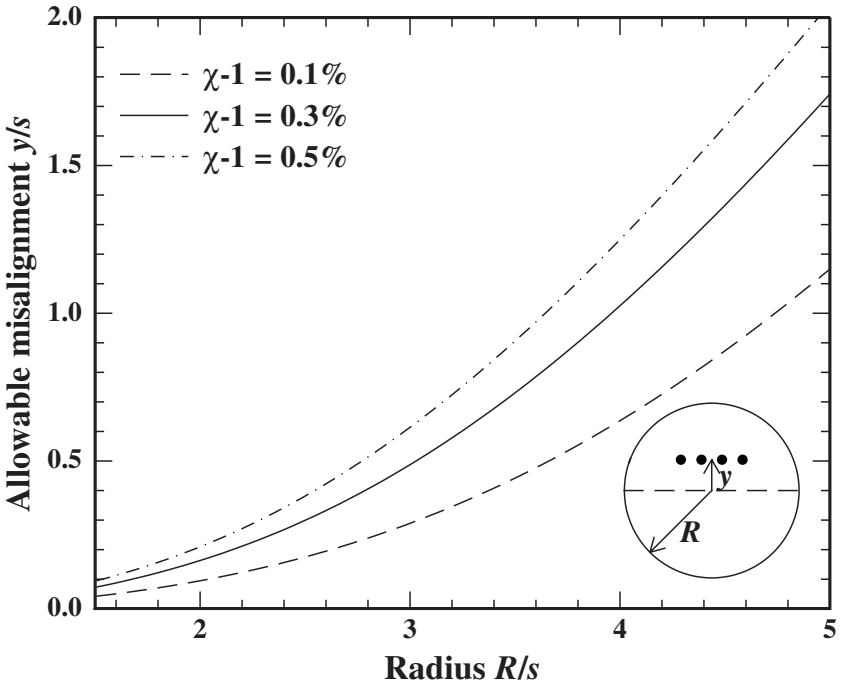

FIG. 11. Allowable misalignment $y / s$ of the four-point probe from the center of a circular disk sample of radius $R$. Calculations are shown for $\chi-1$ $\in[0.1 \%, 0.3 \%, 0.5 \%]$.

$\in[0.1 \%, 0.3 \%, 1 \%, 3 \%, 10 \%, 30 \%]$ are shown. To assist the visual interpretation of the graph the disk perimeter is shown as a full red line, and a sketch of the four-point probe in its ideal center position is added. The shape of the "sweet spot" has a striking resemblance to a cat's eye, and is seen to have a considerable width.

Figure 11 shows the width of the sweet spot as the allowable misalignment $y$ normal to the disk diagonal from the center as a function of the radius $R$ of the circular disk sample. Calculations for allowable errors $\chi-1$ $\in[0.1 \%, 0.3 \%, 0.5 \%]$ are shown. At radii larger than approximately $5 s$ the allowable misalignment $y$ increases approximately linearly with radius, in agreement with the expectation that a certain distance $(\sim 3.75 s)$ from the boundary is required. At smaller radius $(R \leq 5 s)$ a closer relative proximity to the boundary is seen to be allowed.

Figure 12 shows calculated contours relating the intended relative radial position $x / R$ on a diagonal to the relative displacement $y / R$ normal to the diagonal for constant

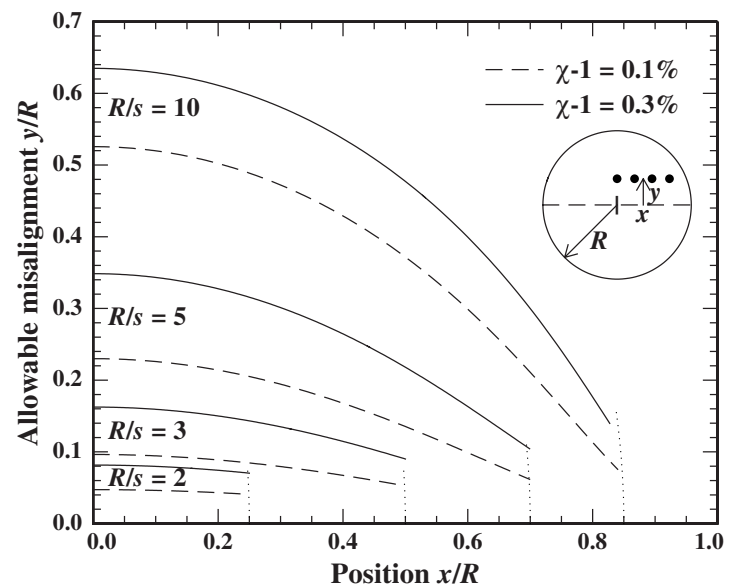

FIG. 12. Relation between relative radial probe position $x / R$ and relative off diagonal position $y / R$ for constant $\chi$ in four-point probe measurements on a circular disk of radius $R$. Calculations for $\chi-1 \in[0.1 \%, 0.3 \%]$ and $R$ $\in[2 s, 3 s, 5 s, 10 s]$ are shown. 


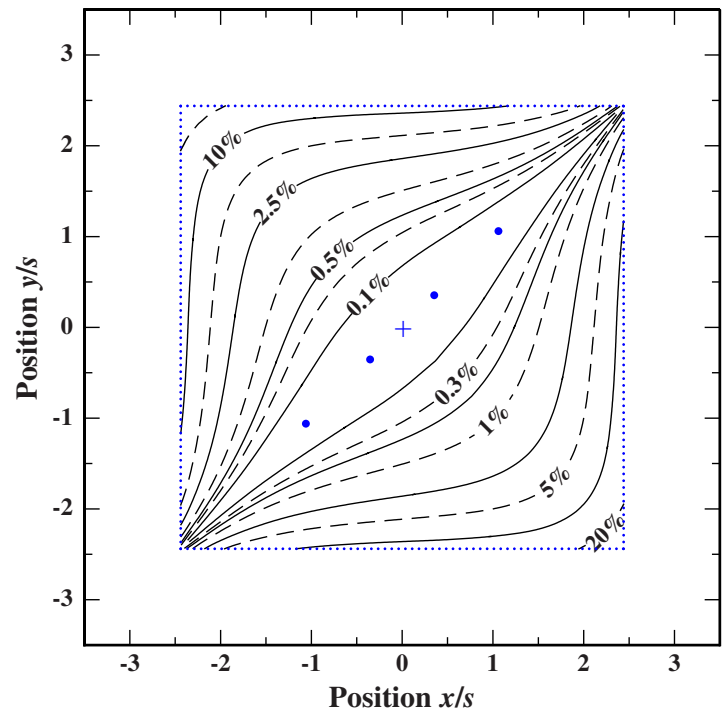

FIG. 13. (Color online) Contour plot showing isoerror curves for the M4PP parallel to the diagonal of a square with the side-width $W=7 \mathrm{~s}$. The dotted square indicates the position of the probe center in the extreme position with one probe pin on the boundary of the square, while the boundary of the square sample coincides with the boundary of the plot. Contour-lines corresponding to $\chi-1 \in[0.1 \%, 0.3 \%, 0.5 \%, 1 \%, 2.5 \%, 5 \%, 10 \%, 20 \%]$ are shown. To assist visual interpretation of the graph the four-point probe is shown in its ideal position.

error $\chi-1 \in[0.1 \%, 0.3 \%]$ calculated using four different values of the disk radius $R \in[2 s, 3 s, 5 s, 10 s]$. The full lines show contours corresponding to $\chi-1=0.3 \%$ while the short dotted curves show the relation between the $x / R$ and $y / R$ in the most extreme position with the outermost probe pin on the boundary of the disk. Figure 12 clearly demonstrates the comparatively large sweet spot on the circular disk, which makes probe alignment very easy experimentally.

\section{E. Square samples}

A square sample has four mirror planes, two along the diagonals and two parallel to the sides. Figure 13 shows isoerror contours (positions of the center of the probe) for a M4PP parallel to the diagonal of a square with the side-width $W=7 \mathrm{~s}$. Calculated contour-lines corresponding to $\chi-1 \in[0.1 \%, 0.3 \%, 0.5 \%, 1 \%, 2.5 \%, 5 \%, 10 \%$, $\times 20 \%]$ are shown as full and dashed lines. The dotted line indicates the trace of the probe center with one probe pin on the edge of the square sample, while the boundary of the sample coincides with the boundary of the plot. The sweet spot is seen to have a considerable width, but it becomes narrower as the probe is moved toward the corner.

Figure 14 shows the allowable transversal misalignment $\delta$ from the ideal position on the middle of the diagonal of a square as a function of the edge length $W$ for three different values of the allowable error $\chi-1 \in[0.1 \%, 0.3 \%, 0.5 \%]$.

Figure 15 shows isoerror contours for a M4PP parallel to an edge of a square sample of width $W=6 \mathrm{~s}$. Contour-lines corresponding to $\chi-1 \in[0.1 \%, 0.3 \%, 0.5 \%, 1 \%, 5 \%$, $\times 10 \%, 20 \%, 40 \%, 60 \%, 80 \%]$ are shown as full and dashed lines. The dotted lines are the trajectory of the probe center with one probe pin on the boundary of the sample.

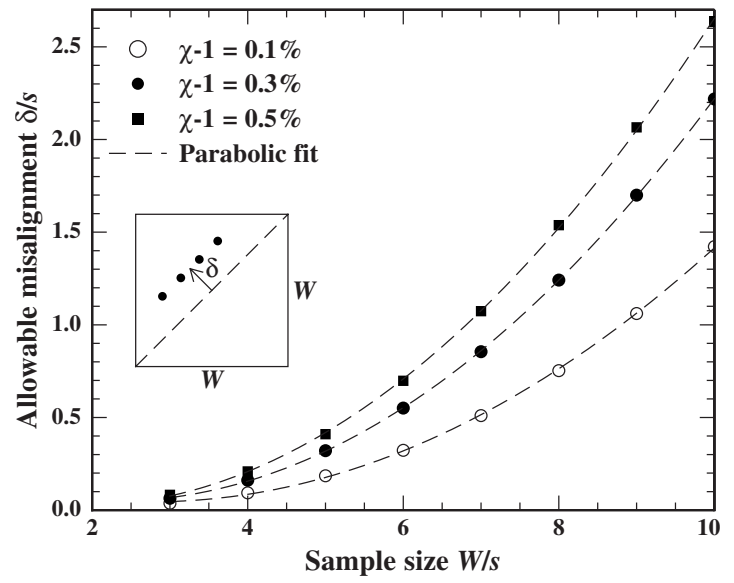

FIG. 14. Allowable misalignment $\delta$ from the ideal diagonal center position on a square sample for the errors $\chi-1 \in[0.1 \%, 0.3 \%, 0.5 \%]$. Calculations are shown for a sample width $W$ in the range from $3 s$ to $10 s$.

The boundary of the plot coincides with the boundary of the sample. The width of the sweet spot is quite large and increases when the probe is moved laterally toward the orthogonal edges. This screening effect is always seen with probes orthogonal to a boundary and is easily understood since images in that boundary contribute significantly to the measured resistance values when the probe is in close proximity to the boundary, and thereby the relative contributions from other boundaries are suppressed.

Figure 16 shows the allowable misalignment $y$ for a probe aligned parallel to one edge of a square sample as a function of the sample size. For square samples larger than $\sim 8 s$ the allowable misalignment increases approximately linearly with sample size in agreement with the expectation that a certain distance $(\sim 3.75 s)$ from a parallel boundary is

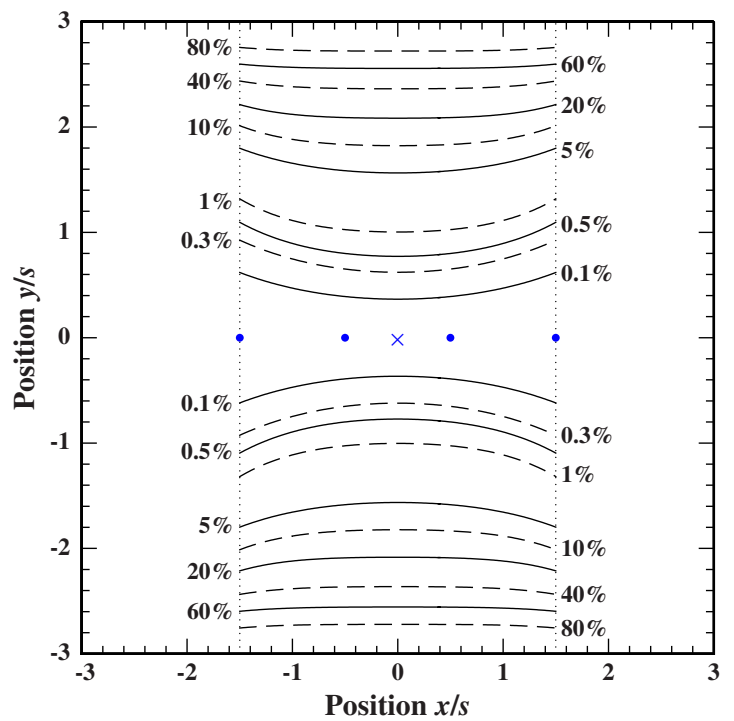

FIG. 15. (Color online) Contour plot showing isoerror curves for the M4PP parallel to an edge of a square sample of width $W=6 \mathrm{~s}$. Contour-lines corresponding to $\chi-1 \in[0.1 \%, 0.3 \%, 0.5 \%, 1 \%, 5 \%, 10 \%, 20 \%, 40 \%$, $\times 60 \%, 80 \%]$ are shown as full and dashed lines. The dotted lines show the trace of the probe center with one probe pin on the edge of the sample, while the sample boundary coincides with boundary of the plot. To assist visual interpretation of the graph the M4PP is shown in its ideal position in the center. 


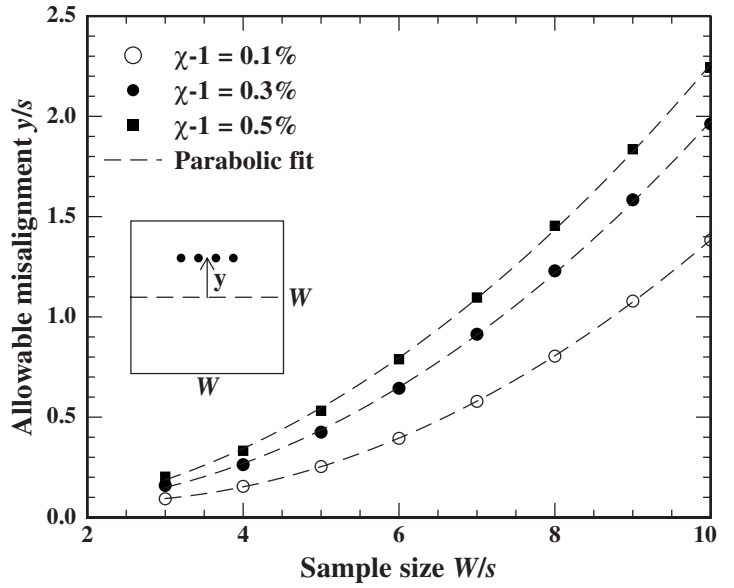

FIG. 16. Allowable misalignment $y / s$ from the ideal position at the center with the probe parallel to the edge of a square sample as a function of the sample size $W / s$. Calculations for $\chi-1 \in[0.1 \%, 0.3 \%, 0.5 \%]$ are shown.

required. For smaller samples a closer relative proximity to the parallel boundary is allowed.

Figure 17 shows error parameter $\chi$ as a function of the probe angle $\theta$ when the probe is placed in the center of a square or rectangular sample; initially, the probe is parallel to the longer edge of the sample. Calculations are shown for squares with $W / s \in[3,3.2,3.5,5]$ and rectangles with $H / W \in[3 s / 3.2 s, 3 s / 3.5 s, 3 s / 5 s, 3 s / 8 s]$. In all cases $\chi$ $\leq 1$ results; it follows that errors due to simultaneous lateral and angular misalignments tend to cancel in the same manner as seen in the case of a narrow stripe sample. For square samples the two types of mirror planes are easily recognized, and the errors due to angular misalignment are small. For instance, the smallest square $(W=3 s)$ where the probe just fits in has $1-\chi \leq 1 \%$, and the error diminishes rapidly with increasing sample size and has almost vanished at $W=5 \mathrm{~s}$. For rectangular samples the two mirror planes are recognized, and the allowable angular misalignment near these two planes differs in the same manner as seen with a narrow stripe; in fact the curve shown for $H / W=3 s / 8 s$ may hardly

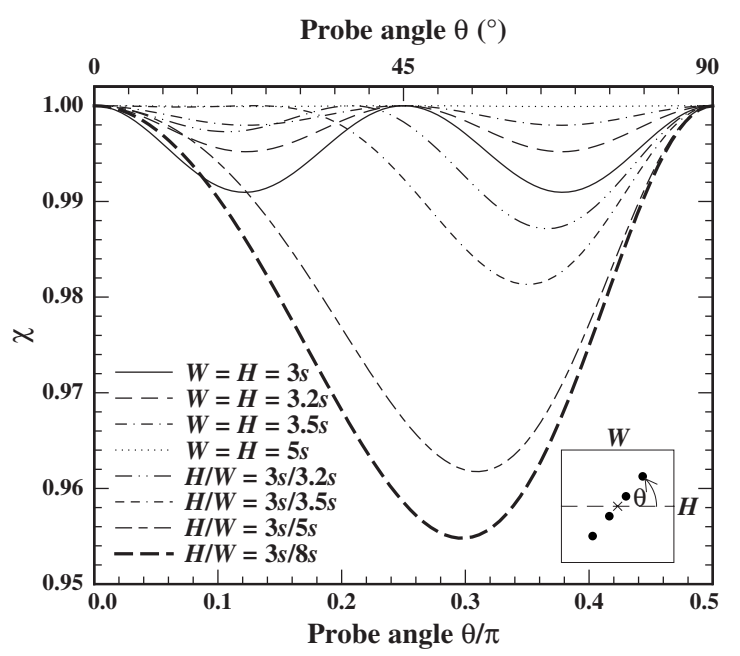

FIG. 17. Error parameter $\chi$ as a function of probe angle $\theta$ with the M4PP positioned at the center of a square or a rectangle. Calculations for squares with $W / s \in[3,3.2,3.5,5]$ and rectangles with $H / W$ $\in[3 s / 3.2 s, 3 s / 3.5 s, 3 s / 5 s, 3 s / 8 s]$ are shown.

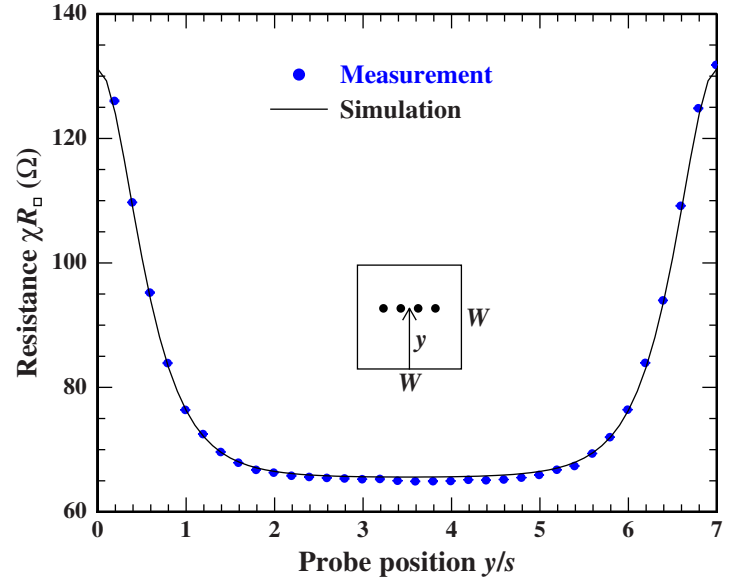

FIG. 18. (Color online) A series of M4PP measurements $(\bullet$ ) on an approximately $70 \times 70 \mu \mathrm{m}^{2}$ sample using a $10 \mu \mathrm{m}$ pitch probe arranged parallel to a sample edge; between each measurement the probe position is incremented by $2 \mu \mathrm{m}$ normal to the line of the probe. The full curve shows model calculations. Excellent agreement between measurement data and model is seen. Note, error bars on the experimental resistance data are drawn, but are not visible.

be distinguished from the corresponding curve for the stripe $W=3 s$ in Fig. 6 .

A comparison of the calculations for the probe aligned parallel to a diagonal and parallel to a side of a square sample, Figs. 14 and 16, respectively, shows that for squares smaller than approximately $8 s$, the allowable misalignment at $0.3 \%$ error is larger for the probe aligned parallel to a side (Fig. 16), thus this represents the preferable measurement configuration on small squares; moreover in this configuration a displacement of the probe in the orthogonal direction increases the allowable misalignment, as seen in Fig. 15. A comparison with the circular disk sample in Fig. 11 reveals that measurements on a square sample parallel to the sides are more favorable than measurements on the inscribed circular disk sample.

\section{Square samples: Experiments}

Figure 18 shows a series of M4PP measurements $(\bullet)$ on a shallow $p$-type Ge junction square pad (approximately 70 $\times 70 \mu \mathrm{m}^{2}$ ) using a $10 \mu \mathrm{m}$ pitch probe, while Fig. 19 illus-

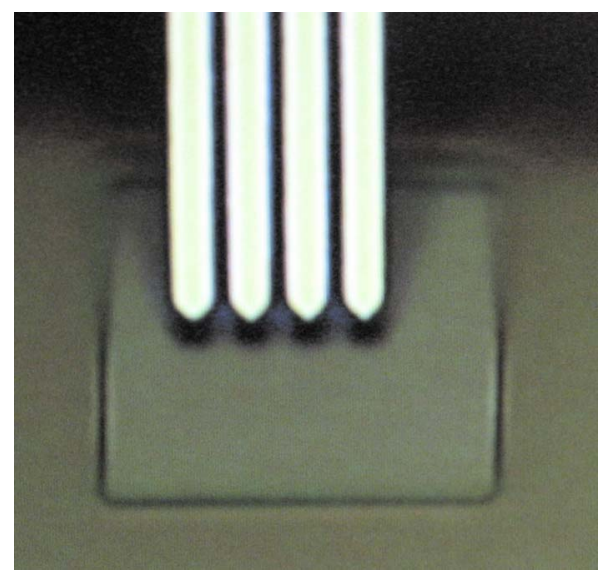

FIG. 19. (Color online) Micrograph showing the $10 \mu \mathrm{m}$ pitch M4PP above a $70 \times 70 \mu \mathrm{m}^{2}$ pad as seen on the screen of the measurement system. 


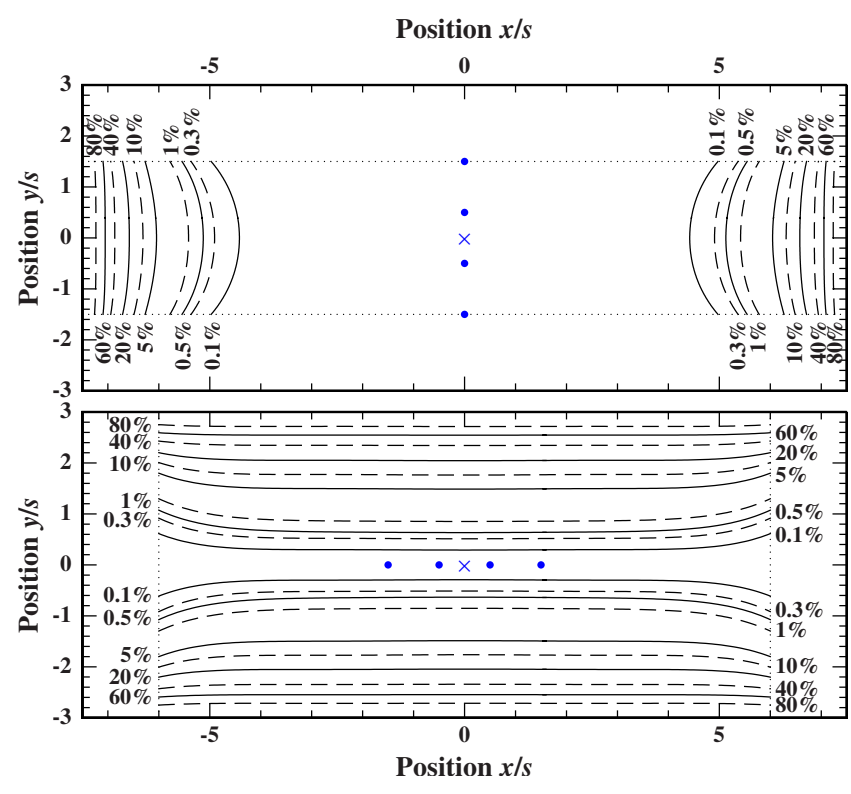

FIG. 20. (Color online) Contour plots showing isoerror curves for a probe arranged parallel to the short (top panel) and long (bottom panel) edge of a rectangle of width $W=15 s$ and height $H=6 s$, respectively. The boundary of the plots coincides with the sample boundary. The dotted lines show the trajectory of the probe center with one probe pin on the boundary of the sample. To ease visual interpretation of the plots the four-point probes are shown in their ideal positions in the center of the sample. Calculations for $\chi-1 \in[0.1 \%, 0.3 \%, 0.5 \%, 1 \%, 5 \%, 10 \%, 20 \%, 40 \%, 60 \%, 80 \%]$ are shown.

trates the probe positioned above the sample prior to measurements. The measurements in Fig. 18 were obtained with the probe aligned parallel to one edge of the sample while it was scanned in steps of $2 \mu \mathrm{m}$ in a direction normal to that edge between each measurement. The full curve shows a model fit to the experimental data and excellent agreement between measurement and model is seen; the small discrepancy in the central part of the scan is most likely due to sample inhomogeneity. The error bars on the measured resistance data are invisible since the relative error is $\leq 0.1 \%$. Error bars on the position are not drawn, but the absolute error on the $2 \mu \mathrm{m}$ step length is less than $50 \mathrm{~nm}$, while the error on the absolute position of the first point relative to the sample edge is significantly larger.

\section{F. Rectangular samples}

A rectangular sample has two mirror planes through the center, one parallel to the short and one parallel to the long edge. Figure 20 show a contour plot for the probe aligned parallel to the short (top panel) and the long (bottom panel) edge of a rectangle of width $W=15 s$ and height $H=6 s$, respectively. The boundaries of the plots coincide with the sample boundaries and the dotted lines show the traces of the probe center with one probe pin on the sample boundary, while the full and dashed lines are the isoerror contours. Calculations for $\chi-1 \in[0.1 \%, 0.3 \%, 0.5 \%, 1 \%, 5 \%$, $\times 10 \%, 20 \%, 40 \%, 60 \%, 80 \%]$ are shown. A significantly larger sweet spot is observed for the probe aligned parallel to the short edge, since again a certain minimum distance from a parallel boundary is required. The larger sweet spot is, however, accompanied by a smaller allowable

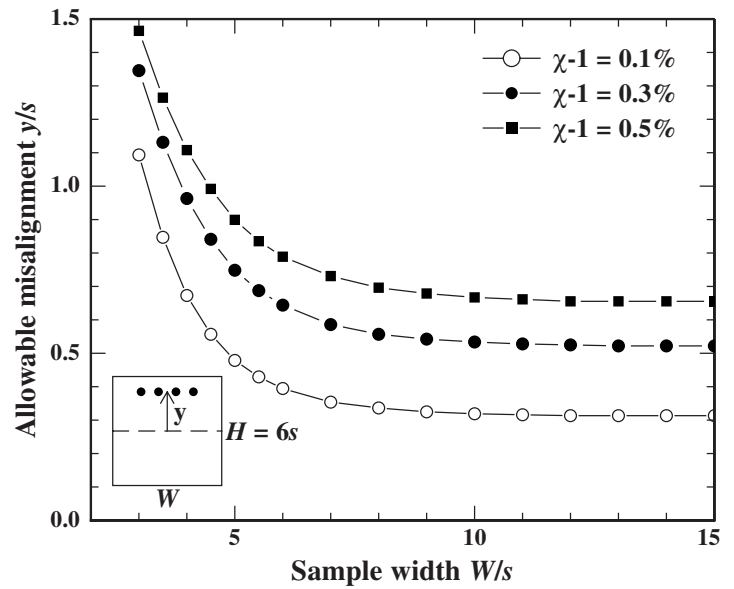

FIG. 21. Allowable misalignment for a M4PP arranged parallel to the edge of width $W$ for a rectangle of height $H=6 s$. Calculations are shown for $\chi$ $-1 \in[0.1 \%, 0.3 \%, 0.5 \%]$ and sample width $W$ in the range from $3 s$ to $15 s$.

angular misalignment as seen in Fig. 17, but still this is the better configuration in practical measurements.

Figure 21 shows the allowable misalignment $y / s$ from the center as a function of sample width $W$ for a rectangle of height $H=6 s$ in the case where the probe is parallel to the edge of length $W$. Calculations for $\chi-1$ $\in[0.1 \%, 0.3 \%, 0.5 \%]$ are shown. Interestingly, the allowable misalignment increases as the sample width decreases due to screening from the boundary orthogonal to the probe. The sample width becomes unimportant when the sample width increases above $W \sim 10 s$, i.e., to the probe the sample seems infinitely wide. It follows that on rectangular samples measurements may advantageously be performed with the probe parallel to the shorter edge.

\section{G. Practical measurement issues}

Currently, M4PPs are available with an electrode pitch in the range of $0.75-20 \mu \mathrm{m}$, while the commonly used probes have an electrode pitch of $10 \mu \mathrm{m}$. How accurately these probes may be positioned depends on the measurement system parameters. Currently, with the CAPRES microRSPM150 system the positioning accuracy is limited primarily by the built-in imaging system, which is inclined at an angle of $60^{\circ}$ to the surface. Therefore a practical, conservatively estimated, positioning accuracy better than $\pm 2.5 \mu \mathrm{m}$ results, while the angular error to some extent depends on the structure of the sample, however, often an error less than $\pm 2^{\circ}$ apply. Figure 19 shows a $10 \mu \mathrm{m}$ pitch probe imaged above a $70 \times 70 \mu \mathrm{m}^{2}$ square sample as seen on the screen of the measurement system. Both misalignment values may be improved if an imaging system with an axis normal to the sample is added. With these practical limitations we shall discuss the implications for practical measurements on the different simple samples.

In the case of a single insulating boundary a measurement with the probe parallel to the boundary may be performed with the probe $\sim 40 \mu \mathrm{m}(3.75 s+2.5 \mu \mathrm{m})$ from the boundary using a $10 \mu \mathrm{m}$ probe, while a measurement with 
the probe normal to the boundary is easily performed with the probe at any distance from the boundary since the angular accuracy is always sufficient.

For a stripe, a measurement with the probe normal to the boundaries may be performed on a sample with a width slightly larger than $3 s$, i.e., $W \geq 35 \mu \mathrm{m}$ for a $10 \mu \mathrm{m}$ probe, to allow for lateral misalignment. In this case the angular alignment accuracy is sufficient in all cases. With the probe parallel to the boundaries a width of $W \geq 45 \mu \mathrm{m}$ ( $W$ $\geq 55 \mu \mathrm{m})$ is sufficient at an accuracy of $0.3 \%(0.1 \%)$ for a $10 \mu \mathrm{m}$ probe. Also here, the angular alignment accuracy is more than sufficient in all cases.

The circular disk sample has a fairly large area where dual configuration measurements are almost unaffected by the proximity of the boundary, from Figs. 11 and 12 it is seen that for a disk of radius $R \geq 23 \mu \mathrm{m}(R \geq 29 \mu \mathrm{m})$ an accuracy better than $0.3 \%(0.1 \%)$ is achieved independent on the angular misalignment.

Dual configuration measurements on a square pad are best performed with the probe aligned parallel to a boundary. Considering a $10 \mu \mathrm{m}$ probe and Fig. 16, measurements using dual configuration are feasible on a square with a side length of $40 \mu \mathrm{m}(50 \mu \mathrm{m})$ or more, and here our calculations (Fig. 17) show that an angular misalignment is without importance.

Measurements on rectangles are best performed with the probe parallel to the shorter edge where the allowable misalignment increases significantly. To allow for lateral misalignment the short edge should be longer than $35 \mu \mathrm{m}$ as in the stripe case.

\section{CONCLUSION}

We have shown that correction free, accurate sheet resistance measurements may be performed using dual configuration measurements on small samples if the measurement is performed close to mirror planes of the sample. In practical measurements samples with characteristic dimensions $\sim 3 \mathrm{~s}$ $+5 \mu \mathrm{m}$ may be characterized with sufficient care assuming an alignment accuracy of $\pm 2.5 \mu \mathrm{m}$. The preferred sample for accurate measurements is a rectangle or a stripe with the probe aligned normal to the long edges; for such samples a $10 \mu \mathrm{m}$ pitch M4PP may be used to accurately $(0.1 \%)$ characterize a sample with a short edge longer than $35 \mu \mathrm{m}$. If it is essential that the area of the sample surface is minimized a square sample should be chosen; in this case the $10 \mu \mathrm{m}$ pitch M4PP may be used to characterize squares with a side length of $40 \mu \mathrm{m}(50 \mu \mathrm{m})$ with a resulting accuracy of $0.3 \%$ $(0.1 \%)$.

\section{ACKNOWLEDGMENTS}

The authors would like to thank Alessandra Satta and Antoine Brugere for preparation of the Ge sample. We are grateful for the financial support from Copenhagen Graduate School for Nanoscience and Nanotechnology (C:O:N:T), the Danish Research Agency (FTP), and Danish National Advanced Technology Foundation. Center for Individual Nanoparticle Functionality (CINF) is sponsored by The Danish
National Research Foundation. We thank Peter Bøggild for continuous support, encouragement and fruitful discussions.

\section{APPENDIX: FOUR-POINT RESISTANCE SOLUTIONS}

The potential and the four-point resistance in the single straight boundary case is easily calculated using point current sources and mirrors of these current sources. ${ }^{9}$ It is useful to use complex numbers, $z=x+i y$ with $i=\sqrt{-1}$, to represent the coordinates as illustrated in Fig. 22, since that allows use of conformal mapping techniques ${ }^{25}$ for more complicated geometries.

In the single straight boundary case, the $z$-plane in Fig. 22 , the potential due to point current sources $I$ at $z_{p}$ and $-I$ at $z_{n}$ is

$$
\Phi\left(z, z_{p}, z_{n}\right)=\frac{I R_{\square}}{2 \pi} \ln \left|\frac{\left(z-z_{n}\right)\left(z-z_{n}^{*}\right)}{\left(z-z_{p}\right)\left(z-z_{p}^{*}\right)}\right|,
$$

where $z^{*}$ is the complex conjugate of $z$. The four-point resistance is $R_{A}=\left[\Phi\left(z_{2}, z_{1}, z_{4}\right)-\Phi\left(z_{3}, z_{1}, z_{4}\right)\right] / I$, where $z_{j}$ with $j$ $\in[1,2,3,4]$ are the probe pin positions, thus

$$
\frac{2 \pi R_{A}}{R_{\square}}=\ln \left|\frac{\left(z_{2}-z_{4}\right)\left(z_{2}-z_{4}^{*}\right)}{\left(z_{2}-z_{1}\right)\left(z_{2}-z_{1}^{*}\right)} \frac{\left(z_{3}-z_{1}\right)\left(z_{3}-z_{1}^{*}\right)}{\left(z_{3}-z_{4}\right)\left(z_{3}-z_{4}^{*}\right)}\right|,
$$

and since $R_{B}=\left[\Phi\left(z_{2}, z_{1}, z_{3}\right)-\Phi\left(z_{4}, z_{1}, z_{3}\right)\right] / I$ the similar relation for $R_{B}$ is

$$
\frac{2 \pi R_{B}}{R_{\square}}=\ln \left|\frac{\left(z_{2}-z_{3}\right)\left(z_{2}-z_{3}^{*}\right)}{\left(z_{2}-z_{1}\right)\left(z_{2}-z_{1}^{*}\right)} \frac{\left(z_{4}-z_{1}\right)\left(z_{4}-z_{1}^{*}\right)}{\left(z_{4}-z_{3}\right)\left(z_{4}-z_{3}^{*}\right)}\right| .
$$

\section{Narrow stripe}

The conformal mapping pair $z=\exp (\pi w / W)$ and $w$ $=W / \pi \ln z$ maps the stripe $0 \leq \operatorname{Im} w \leq W$ on the upper halfplane $\operatorname{Im} z \geq 0$, as illustrated in Fig. 22. In particular the probe pin positions $w_{j}$ with $j \in[1,2,3,4]$ are mapped into the half plane positions

$$
z_{j}=\exp \left(\pi \frac{w_{j}}{W}\right) .
$$

With these positions the four-point resistances may be calculated using Eqs. (A2) and (A3).

\section{Circular disk}

The conformal Möbius mapping pair $w=R(z-i) /(z+i)$ and $z=i(R+w) /(R-w)$ maps the circular disk $|w| \leq R$ on the

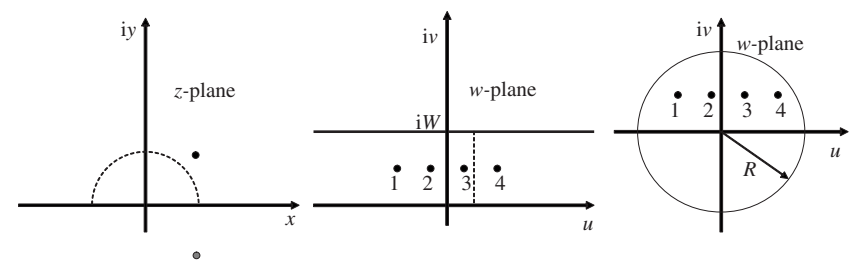

FIG. 22. Left, the upper half plane $\operatorname{Im} z \geq 0$ with an insulating boundary at $\operatorname{Im} z=0$. In the middle, the narrow stripe $0 \leq \operatorname{Im} w \leq W$ with a collinear four-point probe. Right, the circular disk $|w| \leq R$ with a collinear four-point probe. 
upper half-plane $\operatorname{Im} z \geq 0$. In particular the probe pin positions $w_{j}$ with $j \in[1,2,3,4]$ are mapped into the half plane positions

$$
z_{j}=i \frac{\left(R+w_{j}\right)}{\left(R-w_{j}\right)} .
$$

With these positions the four-point resistances may be calculated using Eqs. (A2) and (A3).

\section{Corner}

The conformal mapping pair $z=w^{\pi / \varphi}$ and $w=z^{\varphi / \pi}$ maps the corner $0 \leq \operatorname{Arg}(w) \leq \varphi$ on the upper half-plane $\operatorname{Im} z \geq 0$; and in particular the mapping pair $z=w^{2}$ and $w=\sqrt{z}$ maps the right angle corner $0 \leq \operatorname{Arg}(w) \leq \pi / 2$ on the upper half-plane $\operatorname{Im} z \geq 0$. The probe pin positions $w_{j}$ are mapped onto $z_{j}$ $=w_{j}^{2}$.

\section{Square}

The conformal mapping $w=\int \frac{d z}{\sqrt{z} \sqrt{z^{2}-1}}$ maps the upper halfplane $\operatorname{Im} z \geq 0$ on a square. The integral, however cannot be solved analytically and thus cannot be inverted analytically; it follows that conformal mapping is not as straightforward to apply for evaluation of four-point probing on a square.

${ }^{1}$ D. K. Schroder, Semiconductor Material and Device Characterization, 3rd ed. (Wiley, New York, 2006).

${ }^{2}$ P. Blood and J. W. Orton, The Electrical Characterization of Semiconductors: Majority Carriers and Electron States, Techniques of Physics (Academic, London, 1992), Vol. 14.

${ }^{3}$ C. L. Petersen, T. M. Hansen, P. Bøggild, A. Boisen, O. Hansen, T. Hassenkam, and F. Grey, Sens. Actuators, A 96, 53 (2002).
${ }^{4}$ Capres A/S, http://www.capres.com.

${ }^{5}$ T. Clarysse, A. Moussa, F. Leys, R. Loo, W. Vandervorst, M. C. Benjamin, R. J. Hillard, V. N. Faifer, M. I. Current, R. Lin, and D. H. Petersen, Mater. Res. Soc. Symp. Proc. 912, 197 (2006).

${ }^{6}$ C. L. Petersen, R. Lin, D. H. Petersen, and P. F. Nielsen, Proceedings of the 14th IEEE International Conference on Advanced Thermal Processing of Semiconductors, RTP 2006 (IEEE, New York, 2006), pp. 153-158.

${ }^{7}$ D. H. Petersen, R. Lin, T. M. Hansen, E. Rosseel, W. Vandervorst, C. Markvardsen, D. Kjær, and P. F. Nielsen, J. Vac. Sci. Technol. B 26, 362 (2008).

${ }^{8}$ D. H. Petersen, O. Hansen, R. Lin, P. F. Nielsen, T. Clarysse, J. Goossens, E. Rosseel, and W. Vandervorst, Proceedings of the 16th IEEE International Conference on Advanced Thermal Processing of Semiconductors, RTP 2008 (IEEE, New York, 2008), pp. 251-256.

${ }^{9}$ D. H. Petersen, O. Hansen, R. Lin, and P. F. Nielsen, J. Appl. Phys. 104, 013710 (2008)

${ }^{10}$ F. M. Smits, Bell Syst. Tech. J. 37, 711 (1958).

${ }^{11}$ A. Uhlir, Jr., Bell Syst. Tech. J. 34, 105 (1955).

${ }^{12}$ D. Vaughan, Br. J. Appl. Phys. 12, 414 (1961).

${ }^{13}$ A. Mircea, J. Sci. Instrum. 41, 679 (1964).

${ }^{14}$ M. Albert and J. Combs, IEEE Trans. Electron Devices 11, 148 (1964).

${ }^{15}$ D. Perloff, J. Electrochem. Soc. 123, 1745 (1976).

${ }^{16}$ M. Buehler and W. Thurber, Solid-State Electron. 20, 403 (1977).

${ }^{17}$ L. J. Swartzendruber, Solid-State Electron. 7, 413 (1964).

${ }^{18}$ M. Yamashita and M. Agu, Jpn. J. Appl. Phys., Part 1 23, 1499 (1984).

${ }^{19}$ R. Rymaszewski, J. Phys. E: J. Sci. Instrum. 2, 170 (1969).

${ }^{20}$ D. S. Perloff, J. N. Gan, and F. E. Wahl, Solid State Technol. 24, 112 (1981).

${ }^{21}$ ASTM Standard F1529, (1997).

${ }^{22}$ M. Yamashita, T. Nishi, and H. Mizutani, Jpn. J. Appl. Phys., Part 1 42, 695 (2003).

${ }^{23}$ L. J. van der Pauw, Philips Res. Rep. 13, 1 (1958).

${ }^{24}$ L. J. van der Pauw, Philips Tech. Rev. 20, 220 (1958).

${ }^{25}$ W. Kaplan, Introduction to Analytic Functions (Addison-Wesley, Reading, MA, 1966).

${ }^{26}$ D. Kjaer, R. Lin, D. H. Petersen, P. M. Kopalidis, R. Eddy, D. A. Walker, W. F. Egelhoff, and L. Pickert, AIP Conf. Proc. 1066, 167 (2008). 